\title{
History of rotavirus research in children in Malawi: the pursuit of a killer
}

\author{
Nigel Cunliffe', Desiree Witte, ${ }^{2}$ Bagrey Ngwira ${ }^{2}$ \\ 1.Division of Medical Microbiology, University of Liverpool, UK \\ 2.College of Medicine, Blantyre, Malawi \\ Correspondence author: Dr Nigel A Cunliffe,Division of Medical \\ Microbiology,School of Infection and Host Defence, University of \\ Liverpool,Duncan Building,Daulby Street,Liverpool L69 3GA
}

\begin{abstract}
Rotavirus gastroenteritis is a major health problem among Malawian children. Studies spanning 20 years have described the importance, epidemiology and viral characteristics of rotavirus infections in the country. Despite a wide diversity of circulating rotavirus strains causing severe disease in young infants, a clinical trial of a human rotavirus vaccine clearly demonstrated the potential for rotavirus vaccination to greatly reduce the morbidity and mortality due to rotavirus diarrhoea in Malawi. This new enteric vaccine initiative represents a major opportunity to improve the health and survival of Malawian children.
\end{abstract}

\section{Introduction}

Rotavirus is a leading cause of severe, acute gastroenteritis among infants and young children throughout the world, and is responsible for an estimated 527,000 deaths among children under age 5 years each year1. More than $90 \%$ of childhood deaths attributed to rotavirus infection occur in developing countries, making prevention by vaccination a priority in such settings. This review chronicles the history of rotavirus research in Malawi, from its first identification in the stools of diarrhoeic children to the completion of a large, multi-centre clinical trial of rotavirus vaccine.

\section{How did it all begin?}

In 1987, two Masters of Tropical Medicine students at the Liverpool School of Tropical Medicine (Dr Roberta Pavone and Dr Nicola Schinaia), under the supervision of Professor Tony Hart, Dr Ankie Borgstein and Professor Malcolm Molyneux, undertook a 2 months survey of viral pathogens among children with diarrhoea who attended the under-5 outpatient clinic at the Queen Elizabeth Central Hospital (QECH), Blantyre, Malawi. Using the method of electron microscopy at the University of Liverpool, UK, the characteristic wheel-shaped particles of rotavirus (Figure) were identified in $42 \%$ of 186 faecal specimens examined. The subsequent publication is the first to document rotavirus infection in Malawi (and incidentally, the first to recognise astrovirus infection in Africa)3. The establishment of the Malawi-Liverpool-Wellcome Trust Research Laboratories in Blantyre in the mid-1990s presented Professor Hart with an opportunity to further pursue his interest in rotavirus infections in Malawi. Thus Dr Nigel Cunliffe, under the supervision and guidance of Professor Hart, Professor Molyneux and Professor Robin Broadhead, was awarded a Research Training Fellowship in Clinical Tropical Medicine. His aim was to investigate two aspects of rotavirus disease in Malawi: first, was rotavirus gastroenteritis more severe or persistent in children with HIV infection? Second, what serotypes of rotavirus were circulating?

\section{Study design and methodology}

Dr Roger Glass's laboratory at the Viral Gastroenteritis Section, Centers for Disease Control and Prevention (CDC), Atlanta, Georgia, provided invaluable epidemiological and laboratory support as preparations for study start were finalised. Because rotavirus is most commonly identified among infants with severe gastroenteritis (as opposed to milder disease, were it is detected less frequently), we based our studies at the QECH - a government hospital to which children with severe diarrhoea presented or were referred. A review of hospital records at $\mathrm{QECH}$ had confirmed that diarrhoeal disease accounted for $12 \%$ of all paediatric hospitalisations between 1994 and 19974. Both inpatients and outpatients were enrolled, and faecal and blood specimens were obtained from each child identified with acute gastroenteritis whose parent/guardian agreed to participate. Clinical data were collected by use of a questionnaire and HIV testing was offered to all subjects. Rotavirus antigen was identified using an enzyme immunoassay applied to faecal suspensions. Children with acute rotavirus gastroenteritis with and without HIV infection were followed-up for 3-4 weeks after hospital discharge. Acute and convalescent serum samples were obtained for rotavirus antibody determination (IgG and $\operatorname{IgA}$ ), and faecal specimens were collected at weekly intervals for rotavirus antigen testing. Rotaviruses were genotyped using reverse transcription-polymerase chain reaction (RT-PCR) using consensus and type-specific primers to amplify common rotavirus strain types. Over a thousand children with diarrhoea were enrolled between July 1997 and June 1999, and allowed the two study questions to be answered:

\section{(i) HIV infection did not increase the severity or duration of rotavirus gastroenteritis}

Because infants with congenitalimmunodeficiency syndromes may develop persistent rotavirus diarrhoea with prolonged faecal viral excretion, we hypothesised that this could be a feature of rotavirus infection in HIV-infected infants. Our data indicated, however, that rotavirus disease severity did not differ between infants with and without HIV infection and, while duration of faecal rotavirus shedding appeared increased among HIV-infected infants during short-term follow-up, viral shedding was not associated with diarrhoea5. Moreover, children with and without HIV infection mounted a serum immune response to rotavirus of equal magnitude. Finally, rotavirus did not appear to increase HIV load in plasma6. We considered that these data describing the outcome of wild-type rotavirus infections in HIV-infected infants would encourage the evaluation of rotavirus vaccines in similar populations. Clearly, if rotavirus vaccines were to be used routinely in countries with high prevalence of HIV infection, their safety and efficacy in HIV-infected infants would first need to be demonstrated.

\section{(ii) Rotavirus serotype $G 8$ was apredominant strain type in Blantyre}

Rotavirus is a double-stranded RNA virus whose genome is arranged into eleven segments. Each segment encodes for a viral protein (VP), including six structural proteins and five 
non-structural proteins. The structural proteins together form the mature, triple-layered rotavirus particle. This comprises a core (made up of VP1, VP2 and VP3), which houses the viral genome; a middle layer (VPG) and an outer layer comprising the neutralization antigens VP7 and VP4. The glycoprotein VP7 and the spike protein VP4 form a binary serotyping classification system for rotaviruses, referred to respectively as G-type (for glycoprotein) and P-type (for protease-sensitive protein). In the 1990s, four rotavirus strains predominated globally: these included serotypes G1, G3, and G4 with P[8] VP4 specificity and G2P[4] strains7.

Among the first 100 rotavirus strains characterised in Blantyre in 1997, bothlong and short RNA patterns (electropherotypes) were visualized following polyacrylamide gel electrophoresis and silver staining, indicating some diversity among circulating strains. While long electropherotype strains typed as G3 or G4 using RT-PCR, the majority of short electropherotype strains failed to genotype, with the exception of three G9 strains (G9 was a globally emerging serotype around the turn of the last century)8. To further investigate, the VP7 genes of a few short electropherotype, G-nontypeable rotaviruses were subject to nucleotide sequencing. To our surprise, the nucleotide sequences grouped with those of serotype G8 strains in the Genbank sequence database. An additional primer was designed to enable RT-PCR detection of G8 strains in our collection. Upon repeat examination, the majority of previously G- nontypeable strains typed as G8, and possessed P-types of P[6] and P[4]9. While serotype G8 was a recognised rotavirus serotype of animals (especially cattle), and had sporadically been reported in humans, this was the first time that G8 had been reported as a dominant strain type in children (G8 comprised 51\% of the first 100 strains examined). Incredibly, in a collection of over 400 rotaviruses spanning two years of study in Blantyre (1997-1999), serotype G8 comprised over a third of all strains characterised10. Further molecular and serological characterisation of representative G8P[6] and G8P [4] strains undertaken with collaborators at CDC Atlanta, Akita University, Japan and the National Institute of Health, USA, suggested they were likely human-bovine reassortant rotaviruses, indicating the possibility of an animal reservoir and the occurrence of interspecies rotavirus transmission 11. Since P 6] was better known as a neonatal rotavirus strain type (neonates can excrete rotavirus, but typically without symptoms), we then initiated a study in the neonatal nursery at QECH. A quarter of neonates (without diarrhoea) had rotavirus detected, and each was of the G8P[6] genotype12. Thus while rotaviruses obtained from neonates were generally considered to be distinct from community strains, some overlap was becoming increasingly recognised.

\section{Surveillance of rotavirus gastroenteritis in Blantyre, 1997-2007}

Given the local importance of rotavirus and the unusual strain types that were circulating in Blantyre, we continued to examine rotavirus infections in children at the $\mathrm{QECH}$ for a further eight years. The emphasis was primarily on rotavirus strain characterisation, but these extended studies also allowed us to make several observations regarding the disease burden and epidemiology of rotavirus infections13. First, we have documented that rotavirus is responsible for a third of outpatient attendances and inpatient admissions for gastroenteritis at the $\mathrm{QECH}$, representing a very substantial burden of disease. Second, three-quarters of rotavirus gastroenteritis cases occurred in infants under one year of age, and a third of cases occurred in infants under age 6 months, emphasising the young age distribution of rotavirus gastroenteritis that is typical of developing countries14. Third, rotavirus circulates year-round in Blantyre but with higher rates of detection during the dry \& cooler months of May - July, consistent with rotavirus seasonality patterns observed in other locations in Africa.15 Finally, detailed characterisation of rotavirus strains over this prolonged time period has confirmed that Blantyre is home to a quite remarkable diversity of strain types including G1, G3, G4, G8 and the globally emergent serotypes G9 and (latterly) G1213. Notably, G8 persisted throughout the study period and $\mathrm{P}[\mathrm{G}]$ comprised over a third of all single, fully typeable strains. Multiple G/P combinations were recognised. Taken together, these data suggest that viral reassortment (exchange of RNA segments between co-circulating strains) and interspecies rotavirus transmission have contributed very substantially to a rich diversity of rotavirus strains in Blantyre.

\section{Rotavirus surveillance studies in Lilongwe, Mangochi and Karonga}

Although the major focus of rotavirus research in Malawi has been Blantyre, it was considered important to investigate rotavirus infections elsewhere in the country to see if any regional differences in epidemiology could be determined. A two-year study conducted in collaboration with Dr Charles Mwansambo and Dr Peter Kazembe at Kamuzu Central Hospital, Lilongwe (2005-2007) identified rotavirus in 38\% of children with severe gastroenteritis, and similar to studies in Blantyre, a wide diversity of rotavirus serotypes circulated including (predominantly) G1, G8 and G9. Of particular note, serotype G12 was identified in Malawi for the first time16. Rotavirus was also commonly detected in studies of children presenting with diarrhoea to the rural District Hospitals of Mangochi and Karonga; although G1P[8] was the main strain type encountered, serotype G8 was the second most common G-type identified in both settings during 2005-2007 (Cunliffe NA, unpublished data).

\section{Clinical trial of rotavirus vaccine in Blantyre}

The main hope of preventing childhood deaths due to rotavirus infection is through the use of rotavirus vaccines in early infancy, and efforts have focused on the development and evaluation of orally administered, live, attenuated virus vaccines delivered as part of the childhood EPI schedule. Unfortunately, the first licensed rotavirus vaccine, a tetravalent rhesus-reassortant vaccine (RRV-TV) comprising the globally common G-types G1-G4 (RotaShield, WyethLederle, USA), was causally associated with intussusception after introduction in the USA and was withdrawn from the market. Subsequently, clinical trials of RRV-TV in developing countries were controversially halted and this potentially lifesaving vaccine was unavailable to those children in greatest need. Despite this huge setback, the experience with RRV-TV nevertheless demonstrated that a live, oral rotavirus vaccine could prevent severe rotavirus gastroenteritis. Consequently, two new live, oral rotavirus vaccines were developed by major pharmaceutical companies which represented an enormous stride forward in the battle against rotavirus. These included 
Rotarix (GSK Biologicals, Belgium), an attenuated G1P[8] human rotavirus vaccine and RotaTeq (Merck \& co., USA), a multivalent human-bovine reassortant vaccine comprising G-types G1-G4 and P[8]. Large safety and efficacy trials conducted predominantly in the Americas and Europe demonstrated that both vaccines were highly $(>90 \%)$ efficacious in preventing severe rotavirus gastroenteritis, and neither vaccine was associated with intussusception 17 . While many countries quickly began to adopt a universal rotavirus vaccination policy, the absence of efficacy data from Africa and Asia precluded a recommendation for routine vaccination in those regions. The WHO therefore requested testing of both vaccines in representative populations across both continents1.

In October 2005, a Phase III, placebo-controlled multi-centre clinical trial of GSK's G1P[8] Rotarix vaccine, supported by the Rotavirus Vaccine Program (PATH, USA), was initiated in South Africa and subsequently in Malawi. This landmark study represented the first efficacy trial of a modern rotavirus vaccine in Africa. In Blantyre, over 1,700 subjects were enrolled during a 10 months period and were followed up at weekly intervals to one year of age, after receipt of vaccine or placebo at age 6,10 and 14 weeks. A high incidence of severe rotavirus gastroenteritis was observed among infants in Malawi, which the vaccine reduced by a half18. The majority of rotavirus serotypes that circulated during the study period (including serotypes G8, G9 and G12) were not included in the vaccine formulation. This unusual serotype distribution not only reflected the presence of such strains in Blantyre as demonstrated in concurrent surveillance studies13, but also highlighted the ability of this monovalent vaccine to provide cross-protection against diverse circulating rotavirus strains 19. While the vaccine's efficacy against severe rotavirus gastroenteritis was lower in Malawi than observed in South Africa $(77 \%)$, the public health impact of vaccination is likely to be great in Malawi because of the high incidence of severe rotavirus disease recognised during the trial18.

\section{Global rotavirus vaccine recommendation by WHO}

The WHO's Strategic Advisory Group of Experts (SAGE) met in early 2009 to consider the results of the clinical trials in Malawi, South Africa and elsewhere. Based upon these and other data presented, SAGE issued a recommendation that rotavirus vaccine should be incorporated into the EPI schedules of all countries, and strongly recommended vaccine introduction where diarrhoea deaths account for $>10 \%$ of under 5 -year childhood mortality 20 . This global recommendation by WHO represents a major milestone in the fight against rotavirus, and opens up the possibility of rotavirus vaccines being incorporated into childhood immunization programmes in Africa and Asia in the near future. For Malawi and other countries with high rotavirus and diarrhoeal disease burden, the introduction of rotavirus vaccines would represent a much needed intervention. Finally, over 20 years since rotavirus was first identified in Malawi, a tool exists with which to tackle this killer disease.

\section{Acknowledgements}

This paper is dedicated to the memory of the late Professor Tony Hart. We thank all our colleagues and collaborators who have worked with us over many years, and are grateful to the Wellcome Trust, the World Health Organization, GSK Biologicals and PATH for support of the studies reviewed in this paper.

\section{References}

1. World Health Organization. Rotavirus vaccines. Wkly Epidemiol Rec 2007;82:285-96.

2. Cunliffe NA. Viral gastroenteritis in children with and without HIV infection in Blantyre, Malawi. PhD thesis, University of Liverpool, 2001.

3. Pavone R, Schinaia N, Hart CA, Getty B, Molyneux M, Borgstein A. Viral gastro-enteritis in children in Malawi. Ann Trop Paed 1990;10:1520 .

4. Cunliffe NA, Bresee JS, Gondwe J, Hart CA. The epidemiology of diarrhoeal disease in children at Queen Elizabeth Central Hospital, Blantyre, Malawi, 1994-1997. Mal Med J 1998;11:21-25.

5. Cunliffe NA, Gondwe JS, Kirkwood CD et al. Effect of concomitant HIV infection on presentation and outcome of rotavirus gastroenteritis in Malawian children. Lancet 2001;358:550-55.

6. Jere C, Cunliffe NA, Hoffman IF et al. Plasma HIV burden in Malawian children co-infected with rotavirus. AIDS 2001;15:1439-42.

7. Gentsch JR, Woods PA, Ramachandran $\mathrm{M}$ et al. Review of $\mathrm{G}$ and $\mathrm{P}$ typing results from a global collection of rotavirus strains: implications for vaccine development. J Infect Dis 1996; 174: S30-36.

8. Ramachandran M, Kirkwood CD, Unicomb L et al. Molecular characterization of serotype $\mathrm{G} 9$ rotavirus strains from a global collection. Virology 2000;278:436-44.

9. Cunliffe NA, Gondwe JS, Broadhead RL et al. Rotavirus G and P types in children with acute diarrhea in Blantyre, Malawi, from 1997 to 1998: Predominance of novel P[6]G8 strains. J Med Virol 1999;57:30812.

10. Cunliffe NA, Gondwe JS, Graham SM et al. Rotavirus strain diversity in Blantyre, Malawi, from 1997 to 1999. J Clin Microbiol 2001;39:836-43.

11. Cunliffe NA, Gentsch JR, Kirkwood CD et al. Molecular and serologic characterization of novel serotype G8 human rotavirus strains detected in Blantyre, Malawi. Virology 2000;274:309-20.

12. Cunliffe NA, Rogerson S, Dove $W$ et al. Detection and characterization of rotaviruses in hospitalized neonates in Blantyre, Malawi. J Clin Microbiol 2002;40:1534-37.

13. Cunliffe NA, Ngwira B, Dove W et al. Epidemiology of rotavirus infections in children in Blantyre, Malawi, 1997-2007. J Infect Dis: In Press.

14. Bresee JS, Hummelman E, Nelson EAS, Glass RI. Rotavirus in Asia: The value of surveillance for informing decisions about the introduction of new vaccines. J Infect Dis 2005;192:S1-S5.

15. Cunliffe NA, Kilgore PE, Bresee JS, Luo N, Hart CA, Glass RI. Epidemiology of rotavirus diarrhoea in Africa: a review to assess the need for rotavirus immunization. Bull WHO 1998;76:525-37.

16. Cunliffe NA, Ngwira BM, Dove W et al. Serotype G12 rotaviruses, Lilongwe, Malawi. Emerg Infect Dis 2009;15:87-90.

17. Glass RI, Parashar UD, Bresee JS et al. Rotavirus vaccines: current prospects and future challenges. Lancet 2006;368:323-32.

18. Cunliffe N, Kirsten M, Madhi SA et al. Efficacy of human rotavirus vaccine RIX4414 in Africa during the first year of life. Ped Infect Dis J 2009; 28: Abstract P524.

19. Neuzil K, Madhi S, Cunliffe N et al. RIX4414 is protective against severe RVGE caused by diverse rotavirus serotypes during the first year of life in African infants. Ped Infect Dis J 2009; 28: Abstract P596.

20. World Health Organization. Rotavirus vaccination. Wkly Epidemiol Rec 2009;84:232-36 